AIAA 94-2582

Development of

Doppler Global Velocimetry

for Wind Tunnel Testing

Jam es F. M e yers

NASA - Langley R esearch C e r

$\mathrm{H}$ a m p ton, VA

18th AIAA Aerospace

Ground Testing Conference

June 20-23, 1994 / Colorado Spring

Forperm is sion to copy or republish, contact the Am erican Institute of Aeronautics and Astronautics 370 L'En fant Prom enade, S.W., W a shington, D.C. 20024 


\title{
Development of Doppler Global Velocimetry for Wind Tunnel Testing
}

\author{
by \\ James F. Meyers \\ NASA Langley Research Center \\ Hampton, Virginia 23681-0001, USA
}

\begin{abstract}
The development of Doppler global velocimetry is described. Emphasis is placed on the modifications necessary to advance this nonintrusive laser based measurement technique from a laboratory prototype to a viable wind tunnel flow diagnostics tool. Several example wind tunnel flow field investigations are described to illustrate the versatility of the technique. Flow conditions ranged from incompressible to Mach 2.8 with measurement distances extending from 1 to $15 \mathrm{~m}$.
\end{abstract}

\section{Introduction}

Engineers of modern aircraft are faced with many challenges in advancing the state of the art in military and civilian aircraft design. The emphasis in military aircraft design has shifted from the straightline speed of the past, to the maneuverability needed for the multidisciplinary roles desired of modern aircraft. The priority of older civilian aircraft designs to increase passenger capability has be augmented by the modern needs for increased efficiency with less environmental impact.

Likewise, the flow diagnostic tools used in the past are becoming limited. The trial and error days of refining aerodynamic performance by filling down wind tunnel models, as guided by force balance diagnostics, are gone. Today, this approach has become far too expensive, especially since the results only yield the reaction of the model to the flow, not the interaction of the flow with the model. Thus there was little exploitation of flow properties that would improve flight characteristics. Early attempts to investigate flow properties used intrusive probes to measure flow pressure and velocity, disturbing the very quan tities being measured. Later, nonintrusive techniques such as laser velocimetry were added, providing three-component velocity measurements without interferring with the flow being measured. 
The addition of Computational Fluid Dynamics, CFD, has provided designers a powerful tool which is providing unique insights into complex aerodynamics phenomena. The ever increasing computational resources have extended CFD development beyond the ability to experimentally verify the new codes with current point measurement technologies. Thus, instrument developers were challenged to provide global measurement techniques that could provide experimental databases with data densities matching CFD predictions. In response, Adrian proposed the first nonintrusive, laser-based global velocity measurement technique in the mid 1980's: particle image velocimetry. The powerful ability to obtain instantaneous, quantitative planar velocity measurements resulted in its swift acceptance as a new instrumentation standard. It does, however, require major resources in computation hardware, and time to process the acquired data.

In 1991, Komine et $a l^{1}$ presented a new approach to global velocity measurements. This technique - Doppler Global Velocimetry - can provide three-component velocity measurements using standard CCD video cameras. If this idea can be developed into a wind tunnel flow measurement system, experimental data can be obtained with spatial resolutions comparable to the computational efforts using near realtime acquisition and data processing. Toward this end, NASA Langley Research Center has been conducting an agressive research program to develop this concept into a flow field diagnostics tool. The following is a synopsis of that effort, including example results from several flow field investigations conducted as part of the NASA development program.

\section{Basic Principles}

While the optical arrangement used in Doppler global velocimetry is based on the reference-beam laser Doppler velocimeter developed by Yeh and Cumins, ${ }^{2}$ figure 1 , the innovative method for determining the Doppler shift frequency is based on the use of Iodine vapor as an optical frequency discriminator. ${ }^{1}$ The Iodine absorption line at $514.5 \mathrm{~nm}$ (Argon-ion laser) descends from a loss-less transmission of light to an 80 -percent loss with an optical frequency change of approximately $800 \mathrm{MHz}$, figure 2. If the laser output frequency is tuned to a point midway along the edge of the absorption line, figure 2, collected scattered light from a stationary object or cloud of particles is attenuated by 50 percent as it passes through the Iodine vapor. If the object or particle cloud is moving, the attenuation through the vapor increases or decreases depending on the direction of movement. The change in attenuation is proportional to the magnitude of the Doppler frequency shift of the scattered light as induced by the object's motion along the measurement vector shown in figure 1 . This vector is 
established by the difference between the vector indicating the direction of the scattered light collected by the detector, ô, and the propagation direction of the laser beam, $\hat{1}$. The expected Doppler frequency shift from particles with a velocity $\mathbf{V}$ is expressed by:

$$
\Delta v=\frac{v_{o}(\hat{\mathrm{o}}-\hat{\mathrm{1}}) \bullet \mathrm{V}}{\mathrm{c}}
$$

where $\Delta v$ is the optical shift frequency, $v_{0}$ is the laser output frequency, and $c$ is the speed of light. With the inclusion of an Iodine vapor cell in front of the detector in figure 1, a laser Doppler velocimeter is produced.

During laboratory investigations of this system, Komine et $a l^{3}$ found that this configuration did not work properly. Variations in collected light were more attributable to changes in particle size distribution and number density than optical attenuations in the Iodine vapor induced by velocity. A beam splitter was placed ahead of the Iodine cell to direct a portion of the collected scattered light to a second detector. This detector provided a reference signal that tracked the intensity variations of the collected light. Normalizing the signal detector output by the reference level produced a signal whose amplitude was influenced only by the Iodine vapor.

Further laboratory investigations revealed characteristics that would prove to be the strength of the technique. Since optical energy was the measured parameter, this approach did not require single particle realization as needed by other laser velocimetry methods. Only light levels sufficient to activate the detector were needed, regardless of the scattering source. This characteristic allows velocity measurements to be obtained from extremely small particles, such as water vapor condensation, provided the number densities are sufficient to yield adequate scattered light. A second benefit of measuring optical energy was the ability to use linear detectors such as charged-coupled device (CCD) elements. Thus a collection of CCD elements, such as a video camera, could be used to view a laser light sheet illuminating a cloud of particles to obtain global velocity measurements, figures 3 and 4 . A three-component velocity measurement system could be constructed by adding two additional receivers at different angles to the light sheet, i.e., three different output vectors, $\hat{o}_{a}, \hat{o}_{b}$, and $\hat{o}_{c}$, in equation (1).

\section{The Development of Doppler Global Velocimetry}

The potential of Doppler global velocimetry, DGV, to provide threecomponent validation databases for CFD codes made it an excellent candidate for development into a wind tunnel flow diagnostics tool. The 
added benefit of simplicity in this approach increased the probability that a usable instrument could be constructed without a long gestation period. The following is a synopsis of the effort at the NASA Langley Research Center to move this unique measurement technique from the laboratory to the wind tunnel.

\section{The First One-Component System}

A prototype one-component DGV optical system was constructed based on Komine's original system. ${ }^{1}$ The first laboratory tests measured the velocity of a spinning wheel using a simple analog normalization electronics circuit. The results indicated that minor distortions in the optical system, notably the Iodine vapor cell windows, prohibited exact align ment of the two images. Further, different perspective distortions obtained from three receivers viewing the light sheet from different angles would totally prohibit the overlaying of component velocity images necessary to determine the standard $u, v$, and $w$ velocity components. For example, placing a white card with evenly spaced dots in the measurement plane yields the images shown in figure 5 . The distortions in the three images can be removed, figure 6 , using the piecewise bilinear warping algorithms outlined in reference 4 . These distortions also showed that simple analog normalization circuits were not adequate. The needed warping corrections could only be performed if the images were acquired using digital frame grabbers. Thus a prototype frame grabber capable of simultan eously acquiring the signal and reference images was constructed.

This system was used to perform the first wind tunnel investigation with DGV. A 75-degree delta wing was installed in the Langley Basic Aerodynamic Research Tunnel, BART, to provide a vortex flow field. This investigation was selected because the velocity structure was known, and the flow contained substantial component velocities. Also, three-component laser velocimetry data were available ${ }^{5}$ to provide a check on the DGV measurements.

Although the laboratory investigation of the technique using the spinning wheel was successful, the wind tunnel investigation was not nearly so. Problems ranged from laser power distribution to video camera interlacing. Following the wind tunnel entry, additional laboratory studies were designed specifically to search for the reasons causing the undesirable characteristics. This effort resulted in several modifications to the optical system, and a change in data processing procedures. The cylindrical lens used to create the light sheet was replaced with a high-speed galvanometer scanner to provide a more uniform intensity within the light sheet. Also a quarter-wave plate was 
placed in the laser beam path to circularize the polarization, which smoothes the angularly dependent Mie scattering ${ }^{6}$ intensity variations. Additionally circular polarization minimizes reflectance variations from the beam splitter, especially with the splitter rotated less than 20 degrees from the receiver optical ax is. ${ }^{7}$ The scattered light collecting zoom and relay lenses were removed, placing the receiver optics system in the boresight configuration. ${ }^{3}$ This modification increased the in tensity of light reaching the cameras, and lessened optical distortions in the acquired images. A ramification of the chosen industry standard RS-170 video cameras requires a modification to the data processing procedure. These cameras use alternate line interlacing to acquire an image frame. Since the even lines (field 1) acquire photons $17 \mathrm{msec}$ before the odd lines (field 2), the velocity structures acquired by the two fields were different. Thus the altered data processing software separates the fields and treats them as separate data images.

Following these changes, the wind tunnel investigation was repeated. This entry produced results that showed several interesting flow structures. The cross flow velocity structure measured at 20.5 degrees angle of attack, figure 7 , indicated that the left vortex was a normal vortex, but the right vortex implied that the flow was a solid body of revolution. The right vortex image may represent the flow structure for the transition from a stable vortex to a burst condition. This hypothesis was supported by the flow visualization results shown in figure 8 . Increasing the angle of attack to 40 degrees produced a burst vortex condition. Flow visualization of burst vortices have indicated that they were chaotic and without form. This assumption was further reinforced by the measure of high turbulence levels with laser velocimetry even though the mean velocity distribution appeared to maintain a spatial structure. ${ }^{5}$ The DGV measurements also showed a structure within the average flow, figure 9 . The finding that each image, representing an average of velocity for $1 / 60$ seconds, contained a spatial structure, figure 10, was unexpected. Since these structures varied greatly from image to image, the high turbulence levels found previously were resolved. The acquired experimental database may represent the first evidence indicating that burst vortices maintain orgainized velocity structures, and further, these spatially coherent structures vary greatly with time.

\section{Development in the Wind Tunnel}

A series of flow field investigations were conducted in a variety of wind tunnels to expand the potential of DGV. Additionally, a detailed look at how the various hardware elements of the system interact with one another was undertaken. The experience gained during the test 
program provided the insight needed to increase measurement fidelity. The needed modifications included changes to both the system hardware and the data processing software.

Hardware modifications included the addition of laser frequency monitoring equipment to insure the proper characteristics of the laser light. A portion of the laser output was directed toward a modified DGV receiver. This receiver was equipped with photodiodes instead of cameras to provide continuous monitoring of the transfer ratio through the Iodine vapor, thus tracking the laser frequency. The signal levels were recorded each time a data image was acquired to provide a measure of the laser output frequency. Single frequency operation of the Argon laser was monitored using a standard optical spectrum analyzer to visually check the longitudinal mode structure. If evidence that another standing wave or longitudinal mode is competing for laser cavity resonance, data acquisition would cease and the laser adjusted to regain single frequency operation.

The role of software in DGV extends beyond the normal processing of acquired data. Software is used to perform hardware-like functions that affect the fundamental characteristics of the acquired data images. The dewarping of the acquired signal and reference images to remove optical and perspective distortions, described above, is an example. Other functions developed as a result of the insight gained during this phase of development include methods to: Remove dark current and background light from the signal and reference images; account for variations in pixel sensitivities; equalize the image transfer functions between the two optical paths in the receiver; aligning images between the signal and reference cameras, and among components; and, minimize the influence of charge transfer noise originating in the CCD cameras.

Although the response of a CCD to light intensity is linear, each pixel element comprising a video camera chip has a unique sensitivity. Variations in sensitivity among the pixels result in amplitude noise in the image. These variations can be removed by calibrating the pixel sensitivities. The detector face is illuminated with flat profile light at two levels. The two resulting images are used to develop the needed linear equations. Using offset and scaling factors, slope and intercept images are constructed which are multiplied and added, respectively, to data images yielding a flat pixel response.

Background illumination, composed of ambient light and stray laser radiation, along with the dark current added by the CCD camera, must be removed to obtain accurate data normalizations. The simplest method to obtain a measure of the background level is to perform a 
standard data acquisition without the presence of seed particles. The acquired signal and reference images are then subtracted from the signal and reference data images, respectively. Further, the background images can replace the intercept images calculated during the pixel calibration described above, since the intercept values are also contained in the background images. Thus flattening the pixel response also removes the influence of background radiation.

Even excluding the effect of the iodine vapor on the collected scattered light, the signal and reference cameras will not yield images of equal amplitudes. The optical transfer through the receiver optical system is affected by the transmission/reflection ratio of the beam splitter, scatter from windows on the Iodine vapor cell, and mismatches in the camera lenses. These differences must be removed to yield a flat ratio response between the acquired signal and reference images. Again the easiest approach is a standard data acquisition of a evenly illuminated white target. Ideally, the normalized image would be uniform, with a value of unity. Actual images will not only deviate from unity, but have variations in amplitude representing optical distortions. With proper scaling, the resulting image is used to flatten and equalize the normalized data images.

The first signal and reference images acquired during a data acquisition cycle are dewarped and spatially cross correlated. This operation checks if tunnel vibrations have misaligned the receiver optical system. The peak of the cross correlation will be located at the proper alignment position. The signal image can be spatially shifted by the calculated offset, and thus align the images for proper normalization. This technique is also used to insure proper alignment among the three receivers for accurate resolution of the normal $u$, $v$, and $w$ velocity components.

It is well known that a photograph taken at $\mathrm{f} 16$ is far sharper than a similar photograph taken at $\mathrm{f} 1.2$. The reason is that $\mathrm{f} 16$ will support higher spatial frequencies than f1.2. A measure of this characteristic is the modulation transfer function, or MTF. Likewise, a CCD video camera has its own MTF. This MTF is not based on optical frequencies, but on leakage of charge from one pixel to another, when there are differences between acquired photons among the pixels. The combined optical and electronic MTF can be determined by analyzing the sharpness of a large black dot on a white background as acquired by the signal and reference cameras. The MTF is important because the acquired data images contain spatial frequencies higher than the measured MTF. These high frequencies occur because a given pixel's charge can be randomly altered as it passes from pixel-to-pixel during the output phase. The high frequency portion of this charge transfer 
noise can be removed by spatially low pass filtering each data image at the system MTF.

\section{Aerodynamic Results from the Development Program}

During the course of the DGV development program, various flow field investigations were conducted in several facilities. These tests included the study of reverse flow from a powered engine model in the Langley 14-x 22-foot Subsonic Tunnel, and supersonic flow fields in the Langley Unitary Plan Wind Tunnel, UPWT. These tests were chosen to help determine the limits in focal distance and velocity of the instrument.

The ability of DGV to be extended to a focal distance of $4.0 \mathrm{~m}$ was tested by mapping the flow about a powered model engine mounted to a semispan model of a large transport aircraft. The aerodynamic objective was to determine the interaction of reverse thrust flow with the free stream. This large flow field required a mosaic of three overlapping 0.5-x 0.5-meter measurement planes to fully describe the flow interactions, figure 11. The only ramification of the increased focal distance was the expected decrease in collected scattered light.

The UPWT high speed investigation consisted of several elements, each designed to study a different aspect of DGV as it applies to supersonic research. First was the flow measurement of the empty test section. This showed that water/ice particles produced by water vapor condensation, estimated to be less than 0.1 microns in diameter, produced sufficient scattered light for DGV operation.

The second element was to determine if the condensation particles were indeed 0.1 microns in diameter. A flat plate was inclined to -15 degrees in the test section, and the flow about the oblique shock measured. The results for a free stream Mach number of 2.5 are shown in figure 12 . The particle lag can be determined by viewing the velocity profile along a single row of pixels in the standard X-Y format, figure 13 . While the velocity lag was only $2 \mathrm{~mm}$, it was greater than expected for the small condensation particles. However, it is well within the expected charge transfer noise limits about the MTF function as overlaid in figure 13. Thus, the limit of velocity tracking fidelity was established in this experiment by the camera MTF, and not particle dynamics.

The final element was designed to determine the ability of DGV to investigate a complicated supersonic flow. The chosen experiment was the vortical flow above a 75-degree delta wing at various angles of attack. The measured streamwise velocity component posed a unique 
problem. In this test, the expected Doppler shift frequency was greater than the measurable range. The solution was to locate the laser frequency along the left edge of the Iodine absorption line, figure 1, and measure the Doppler shifted frequency on the right edge. An example data image for the 95-percent chord location at an angle of attack of 24 degrees, with a free stream Mach number of 2.8, is shown in figure 14 .

\section{Extending DGV to Three Components}

The next step in the development program was the addition of two receiver systems to obtain an instrument capable of simultaneously measuring three velocity components. As with the previous development program, laboratory tests were followed by a wind tunnel investigation to determine the system characteristics.

Again, the laboratory investigation consisted of a rotating wheel study. The three receiver optical systems were placed left, right, and above with a 30 degree angle to the plane of the wheel. The wheel was illuminated with a cone of laser light propagating in the horizontal plane, and inclined by 45 degrees to the wheel. The resulting three velocity images are shown in figure 15. Resolving these measurements in to the standard $u, v$, and $w$ velocity components produced the data images shown in figure 16.

In response to a request from researchers at the NASA Ames Research Center, a three-component DGV measurement system was installed in the Ames 40-x 80-foot Wind Tunnel to investigate the flow from a highspeed jet. The component orientation for the investigation of a highspeed jet flow is illustrated in figure 17. Data acquisition was performed with a high-speed image processing system equipped with six gray-scale frame grabbers set to operate simultaneously. The study provided resolved $u, v$, and $w$ velocity components of a small ( $0.22 \mathrm{~m}$ diameter), hot $\left(700^{\circ} \mathrm{C}\right)$ jet with velocities over $500 \mathrm{~m} / \mathrm{sec}$ at focal distances from $14 \mathrm{~m}$ (side scatter) to $15.5 \mathrm{~m}$ (near forward scatter). ${ }^{6}$ An example velocity image of the $u$ or streamwise component of velocity is shown in figure 18. This test represents the longest focal distances achieved by any three-component laser velocimeter.

\section{Concluding Remarks}

The development of Doppler global velocimetry from an idea to a usable wind tunnel flow diagnostics tool has been presented. A combination of laboratory and wind tunnel investigations produced the insight necessary to modify hardware and software to increase measurement 
accuracy. While the wind tunnel investigations have yielded measurements beyond the capability of other laser velocimetry methods, the limits of the technique have yet to be determined. Efforts to better delineate the measurement capabilities with current and other technologies, and develop a complete error analysis are underway.

\section{References}

1. Komine, H.: System for Measuring Velocity Field of Fluid Flow Utilizing a Laser-Doppler Spectral Image Converter. United States Patent 4,919,536, April 24, 1990.

2. Yeh, Y.; and Cummins, H. Z.: Localized Fluid Flow Measurements with a He-Ne Laser Spectrometer. Applied Physics Letters, vol. 4, no. 10, pp. 176-178, May 1964.

3. Komine, H.; Brosnan, S. J.; Litton, A. B.; and Stappaerts, E. A.: Real-Time, Doppler Global Velocimetry. 29th Aerospace Sciences Meeting, paper AIAA-91-0337, Reno, NV, January 7-10, 1991.

4. Meyers, J. F.: Doppler Global Velocimetry - The Next Generation? AIAA 17th Aerospace Ground Testing Conference, paper AIAA-923897, Nashville, TN, July 6-8, 1992.

5. Meyers, J. F.; and Hepner, T. E.: ,Measurement of Leading Edge Vortices from a Delta Wing Using a Three Component Laser Velocimeter. AIAA 15th Aerodynamic Testing Conference, San Diego, CA, paper AIAA-88-2024, May 18-20, 1988.

6. Mie, G.: Optics of Turbid Media. Ann. Phys., vo1. 25, no. 3, 1908, pp. 377445 .

7. Komine, H.; Brosnan, S. J.; Long, W. H.; and Stappaerts, E. A.: Doppler Global Velocimetry Development of a Flight Research Instrumentation System for Application to Non-Intrusive Measurements of the Flow Field. NASA CR-191490, January 26, 1994. 
Laser Propagation Direction, î

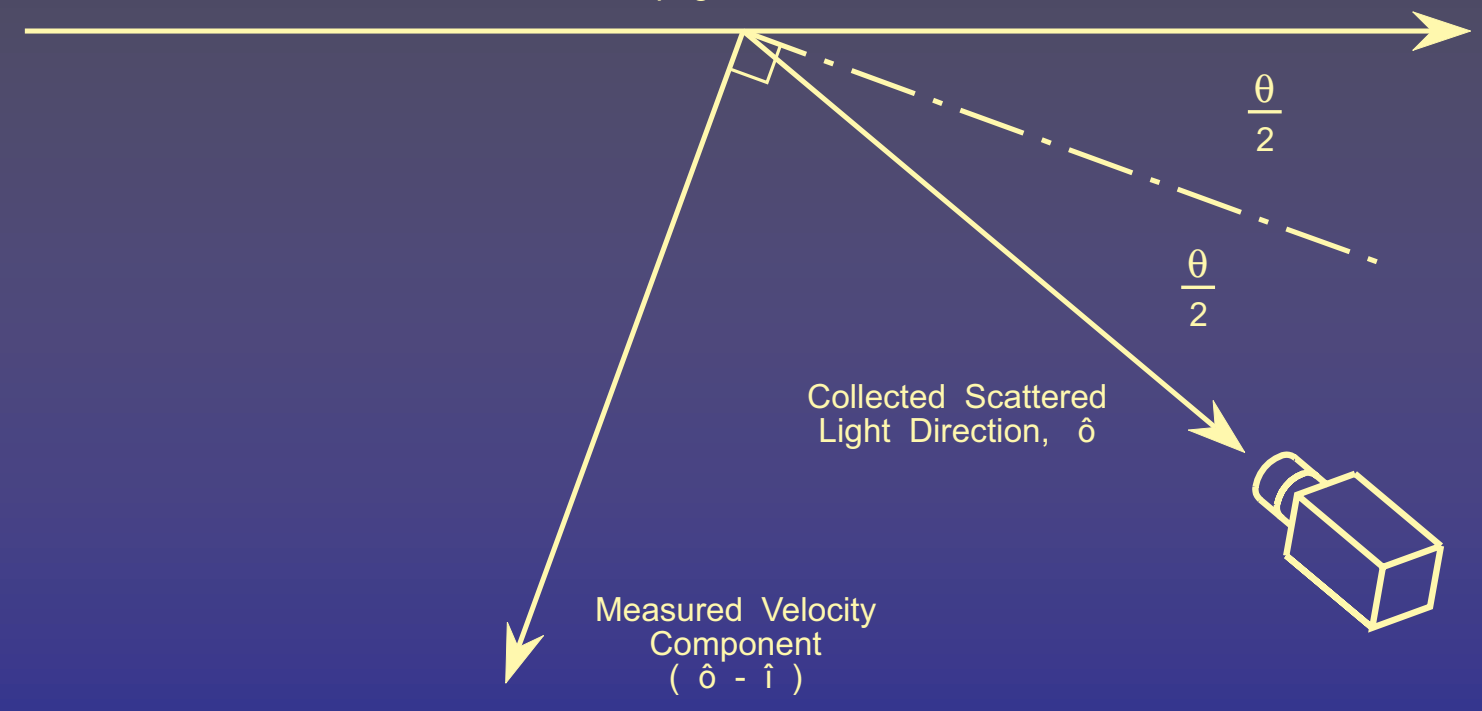

Figure 1.- Diagram depicting the velocity measurement direction based on the orientation of the laser propagation direction and the detector location.

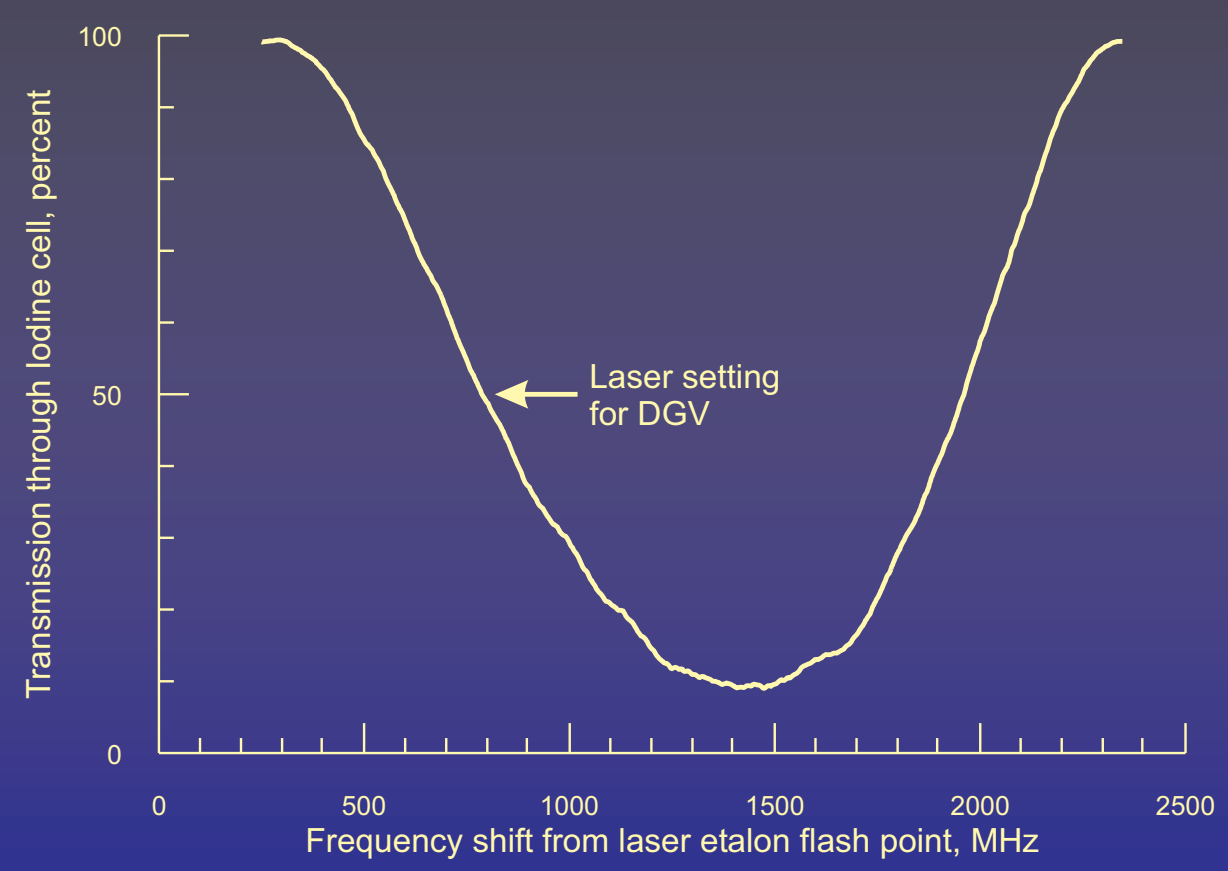

Figure 2.- Transfer function of the Iodine vapor cell, IVC. 


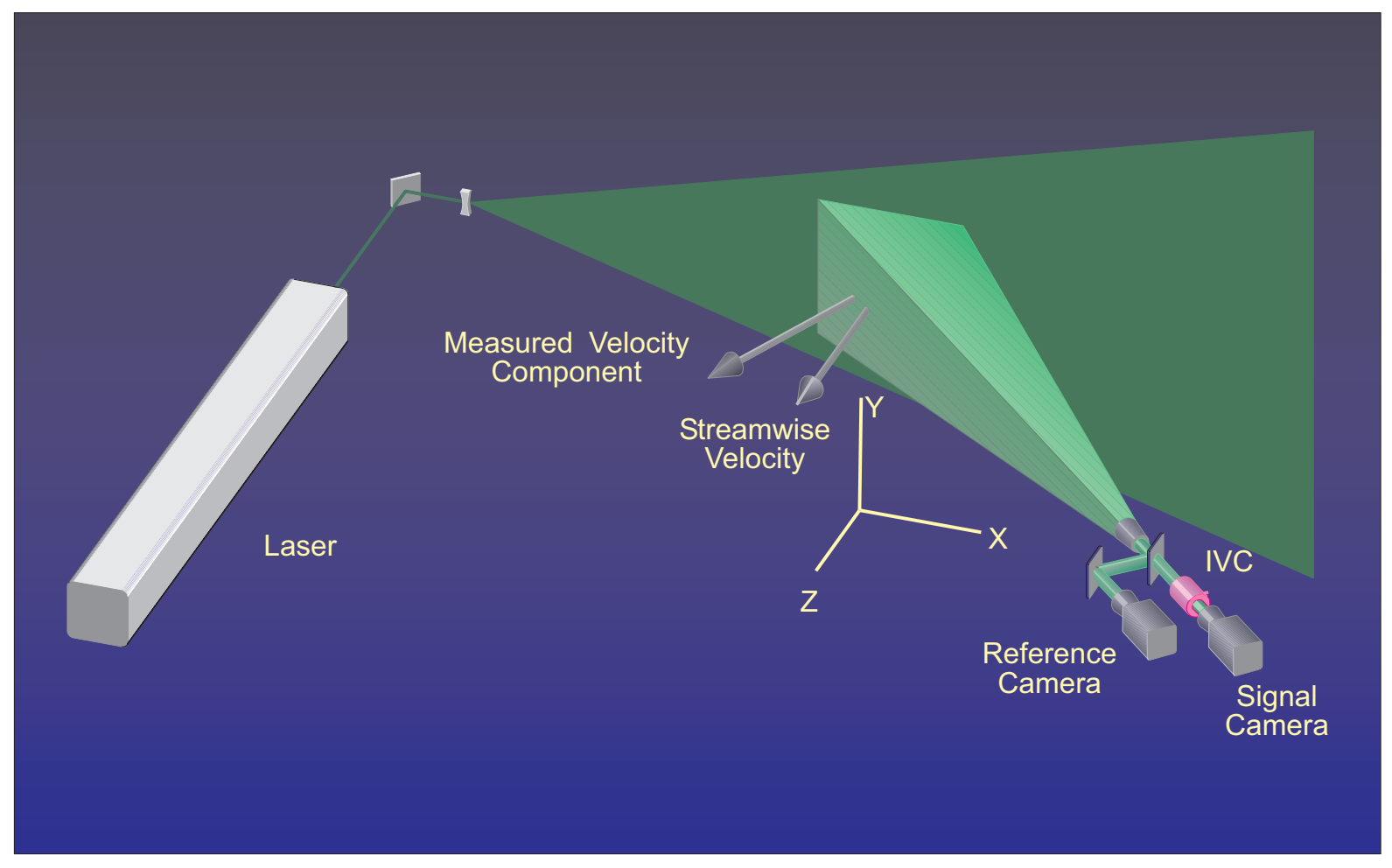

Figure 3.- Pictorial view of the Doppler global velocimeter used in the Basic Aerodynamics Research Tunnel to measure the flow above a 75-degree delta wing.

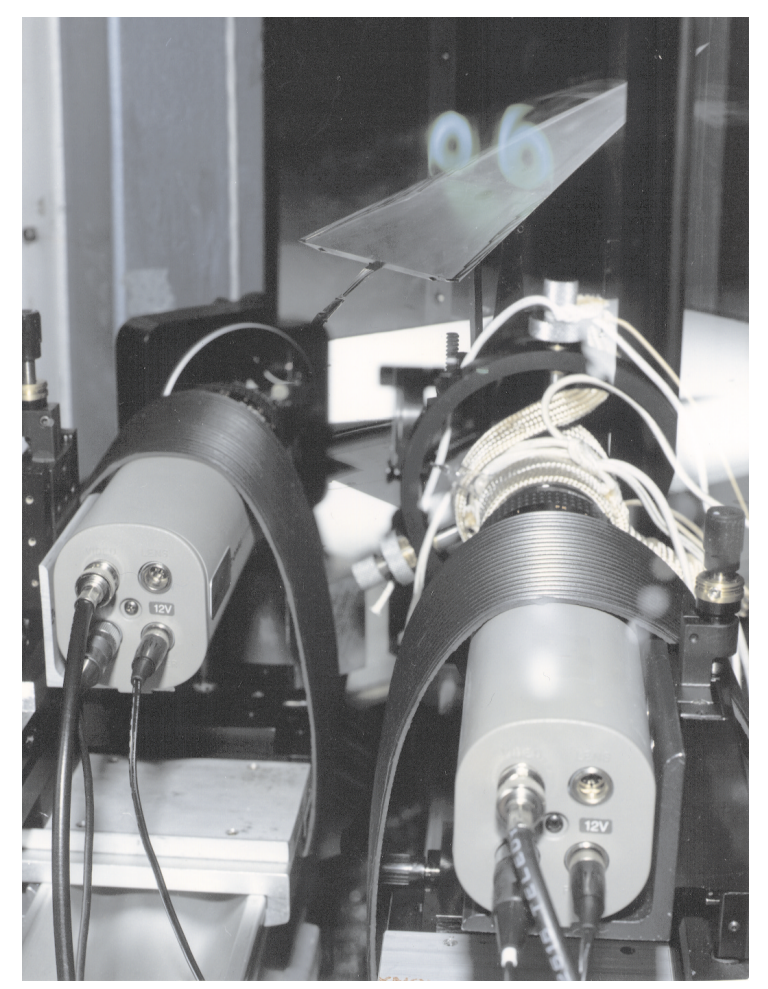

Figure 4.- Photograph of the Doppler global velocimeter installed in the Basic Aerodynamics Research Tunnel. 


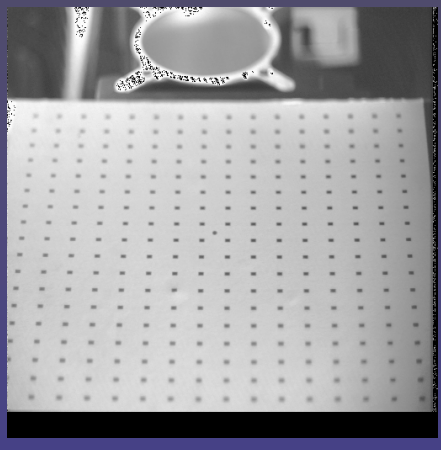

Component A

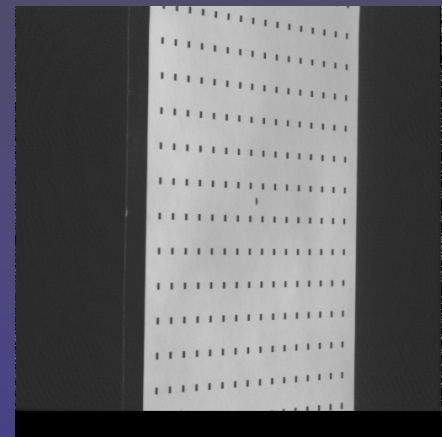

Component B

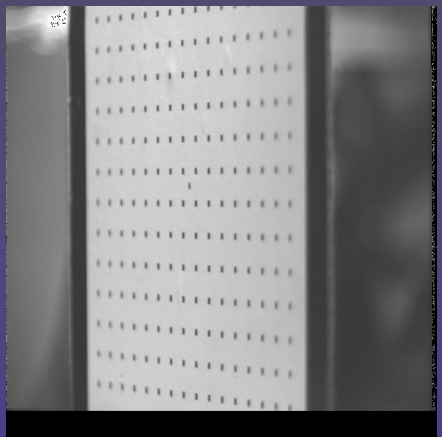

Component C

Figure 5.- Views of equally spaced dots on a flat card from the left, right, and above with a inclination of $30 \mathrm{deg}$ rees from the card plane.

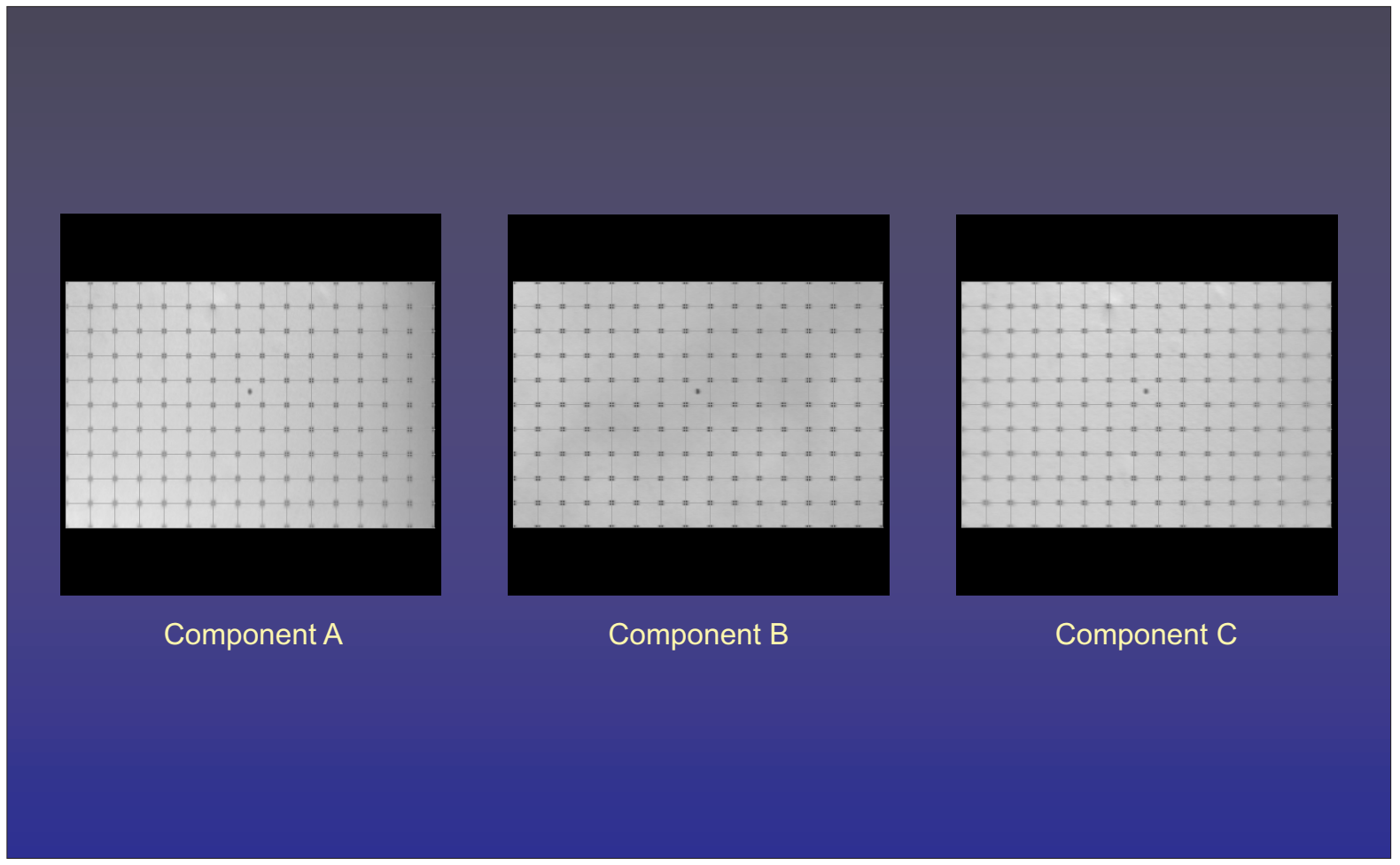

Figure 6.- The views of the flat card shown in figure 5 after warping. 


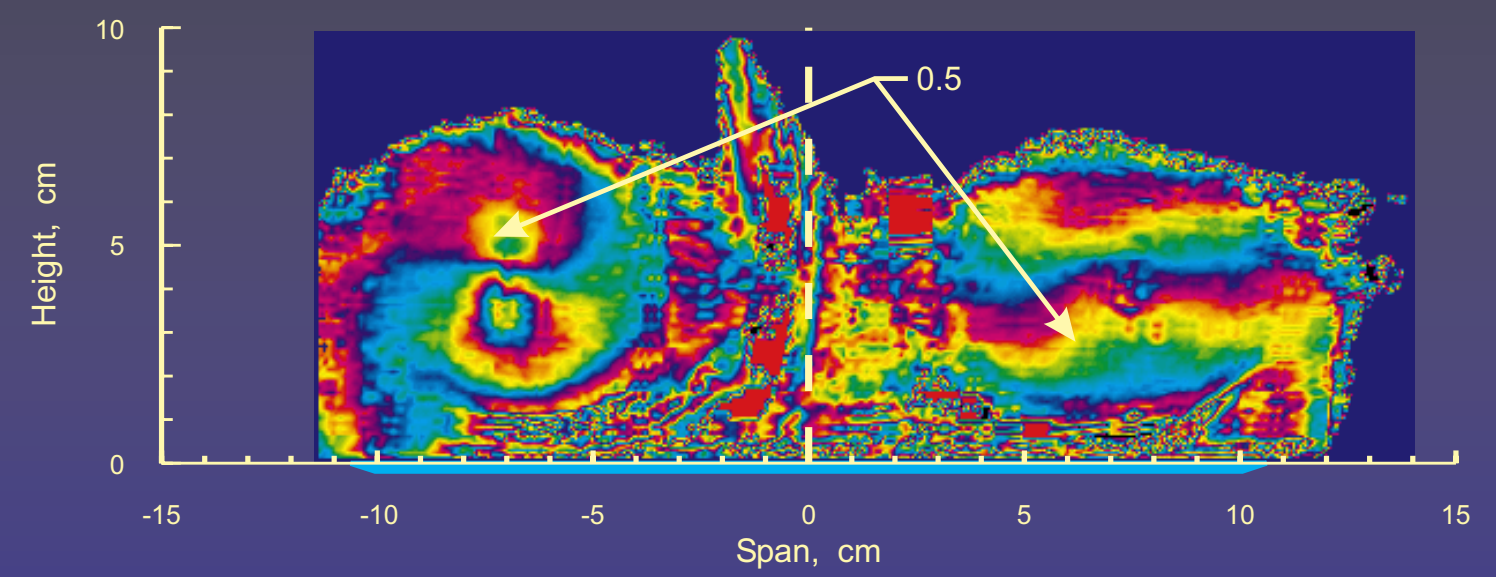

\begin{tabular}{|cccccc|}
\hline & & & & & \\
\hline-0.5 & 0 & 0.5 & 1.0 & 1.5 & 2.0 \\
\hline
\end{tabular}

Figure 7.- DGV measurements of the velocity field above a 75-degree delta wing at an angle of attack of 20.5 degrees, for the component along the direction 71.5 degrees from streamwise in the horizontal plane.

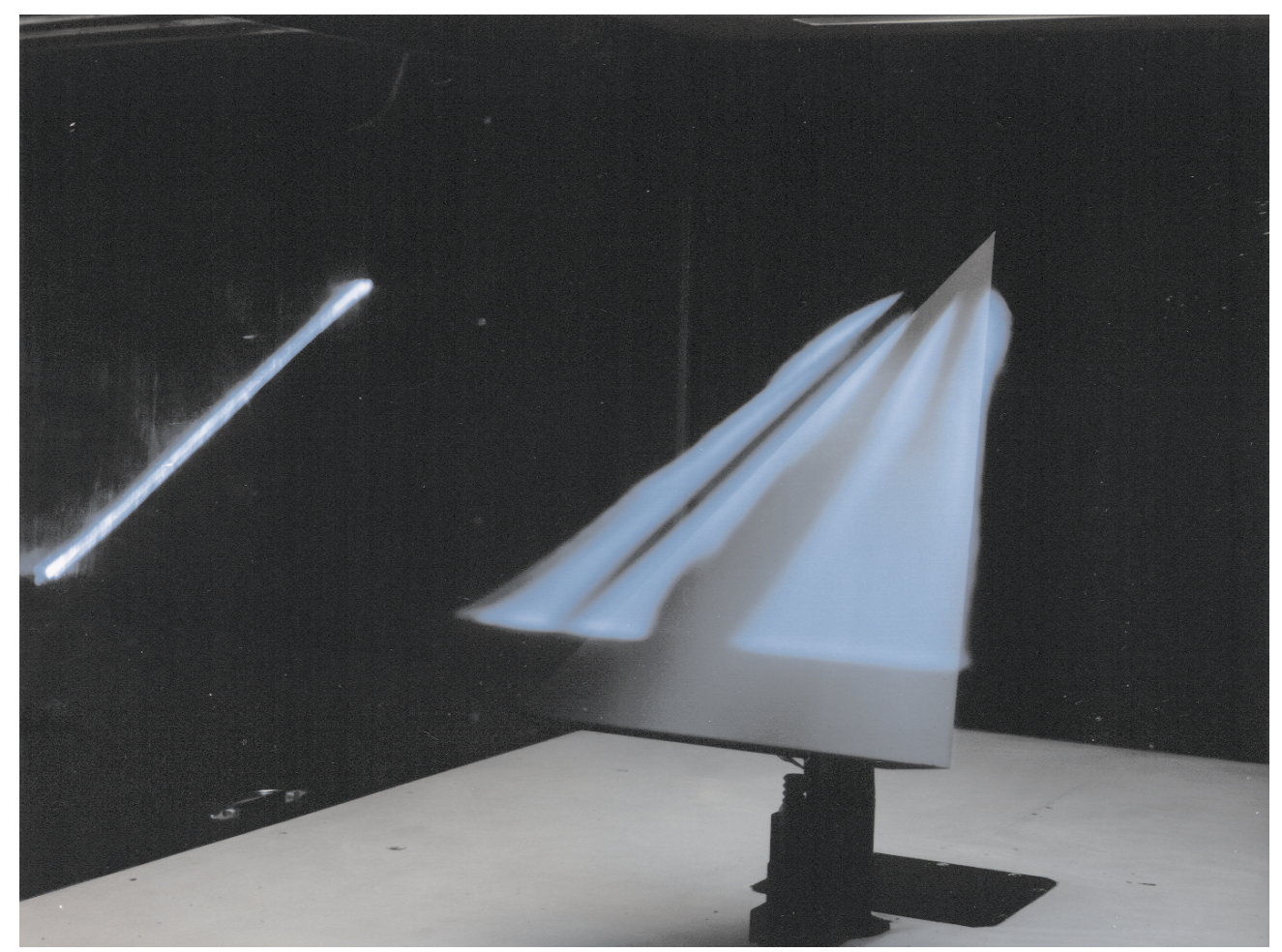

Figure 8.- Flow visualization of the vortical flow above a 75-degree delta wing at an angle of attack of 20.5 degrees. 


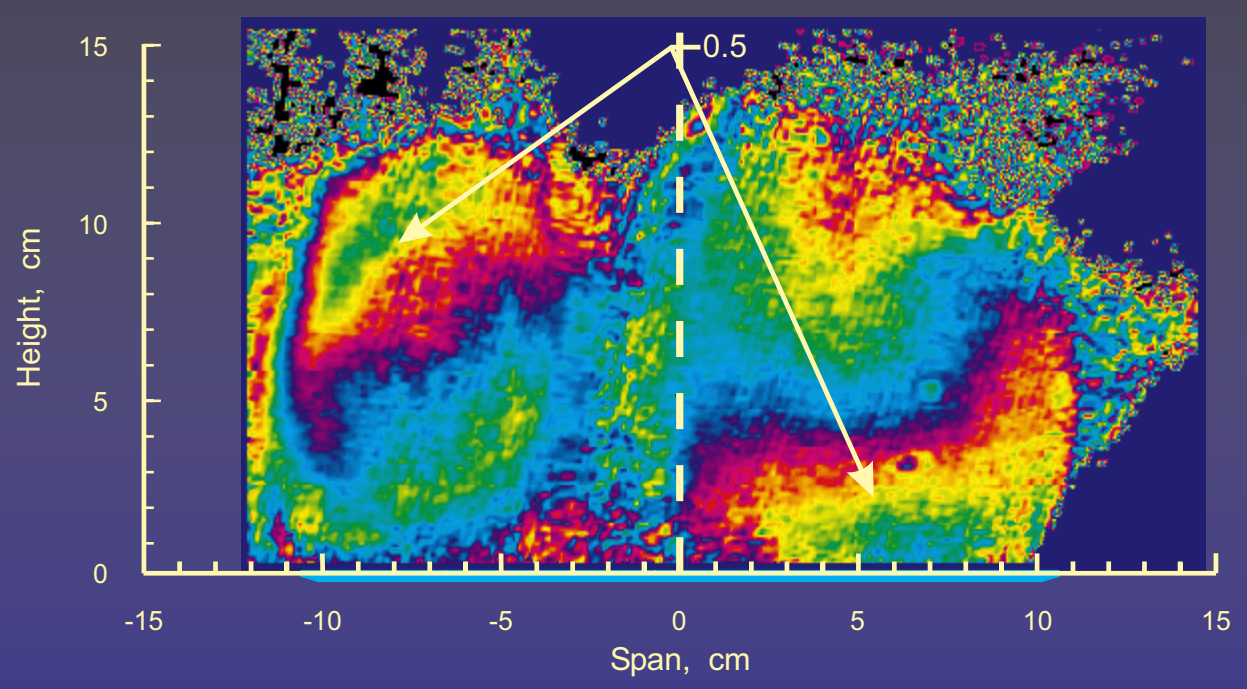

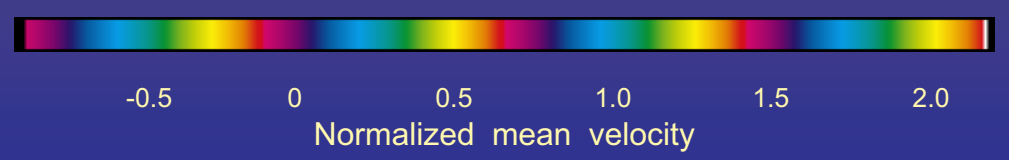

Figure 9.- DGV measurements of the velocity field above a 75-degree delta wing at an angle of attack of 40.0 degrees, for the component along the direction 71.5 degrees from streamwise in the horizontal plane.

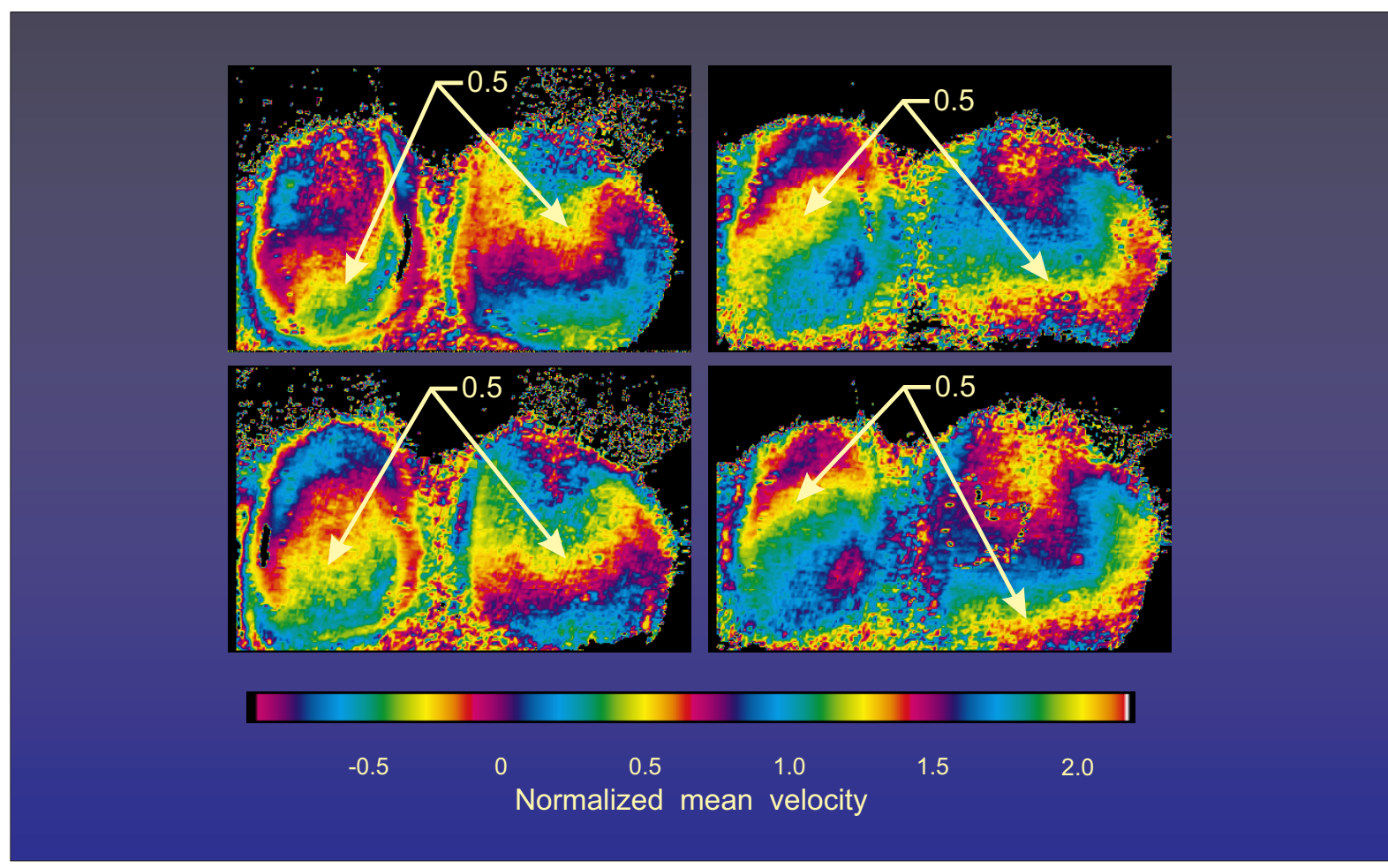

Figure 10.- Individual field images comprising a portion of the data averaged in figure 9 . 


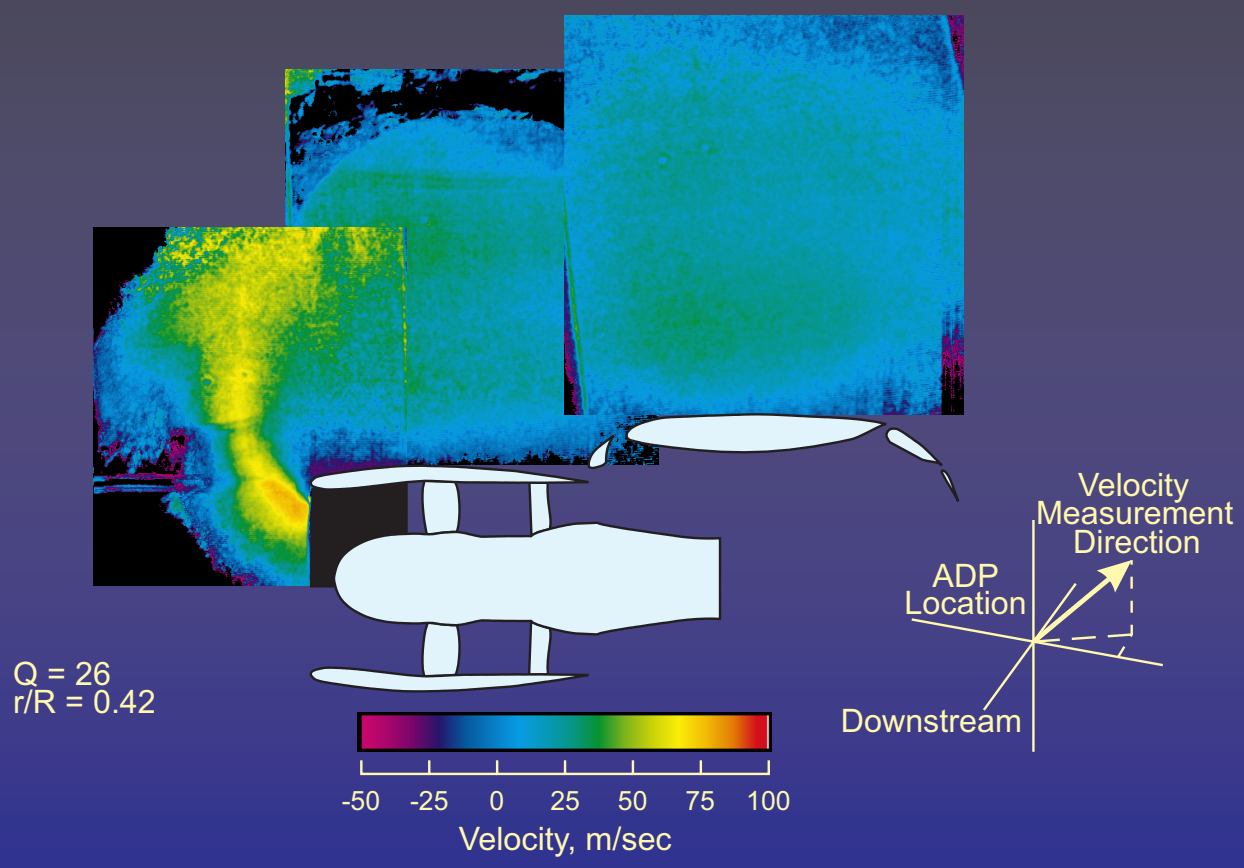

Figure 11.- DGV measurements of jet flow interactions with free stream from an engine operating in reverse thrust.

Contribution of streamwise velocity remains in vertical velocity component

Contribution originates from the change in propagation angle of the scanned laser beam

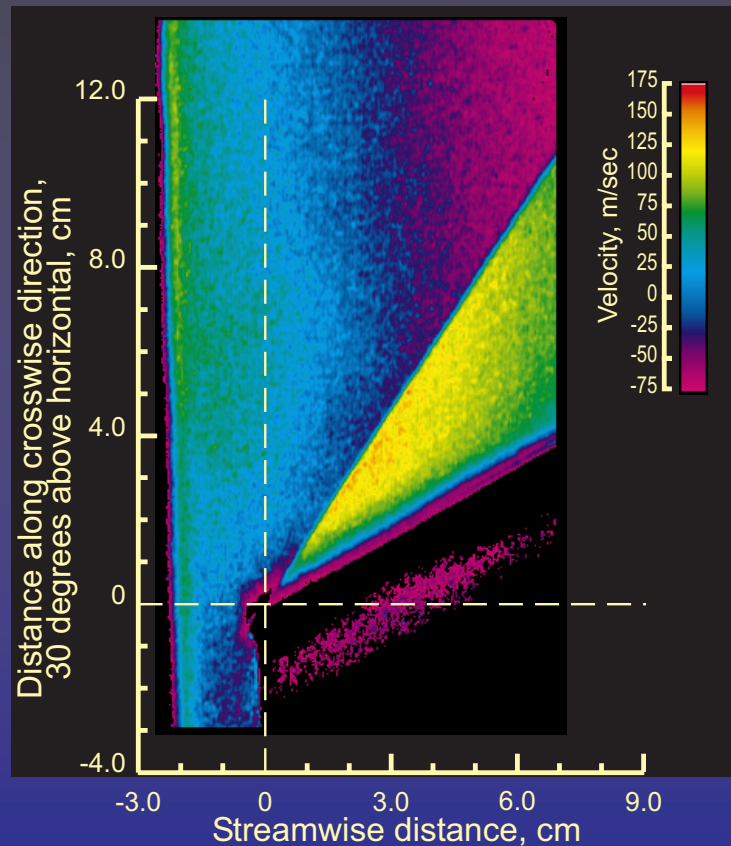

Figure 12.- Map of the vertical velocity component measured by the DGV of the flow above a flat plate inclined to -15 degrees at Mach 2.5. Contributions of the free stream flow are included in the data. 


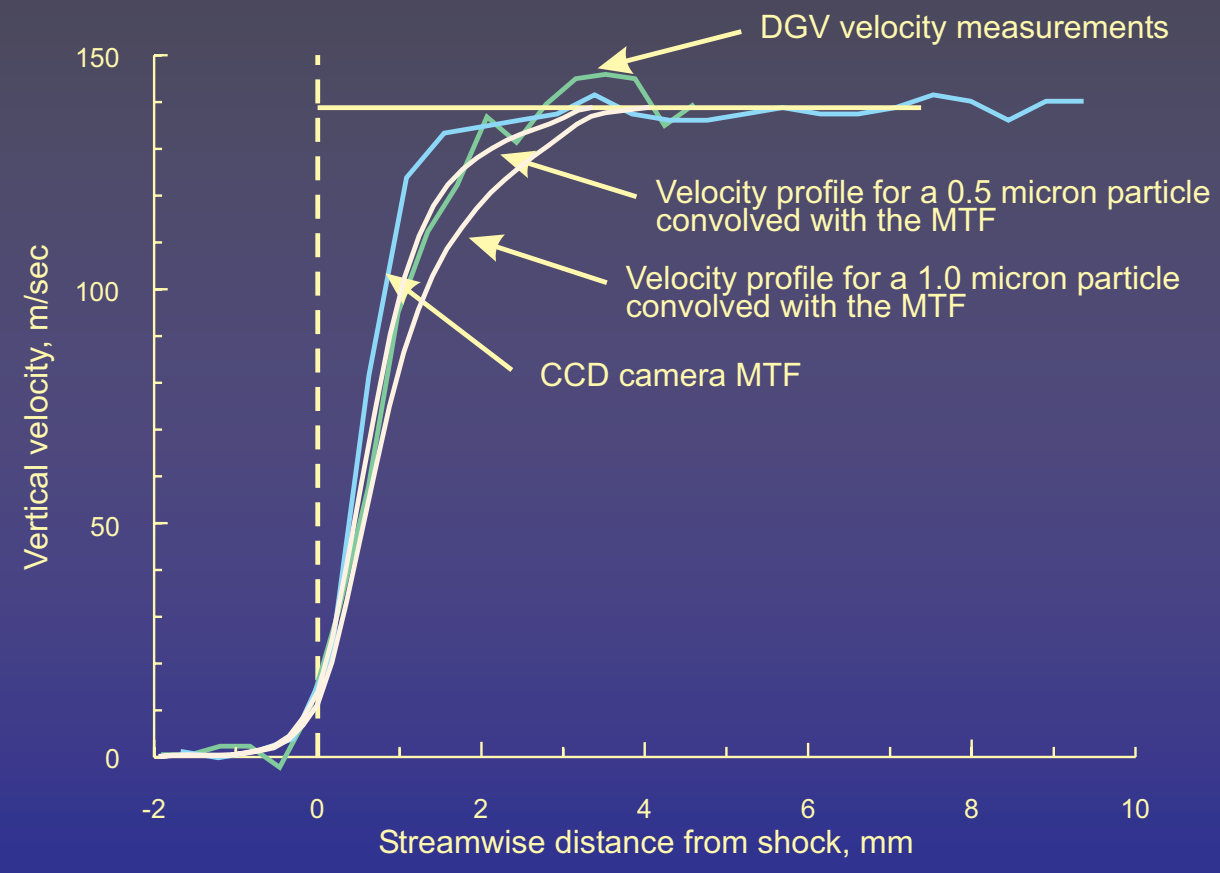

Figure 13.- Comparison of the DGV measurements across the shock with theoretical responses for 0.5 micron and 1.0 micron particles. The CCD camera modulation transfer function indicates the limits of the instrument.

95-percent Chord

Velocity component: $-0.90 i+0.31 j+0.32 k$

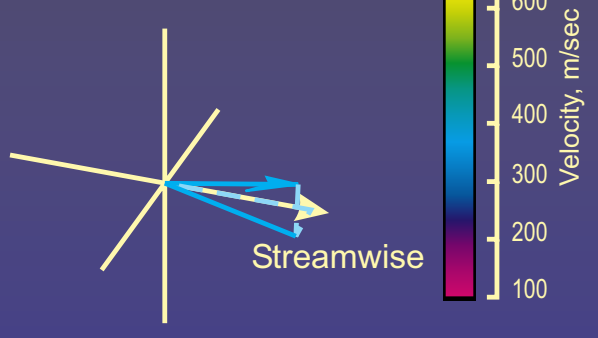

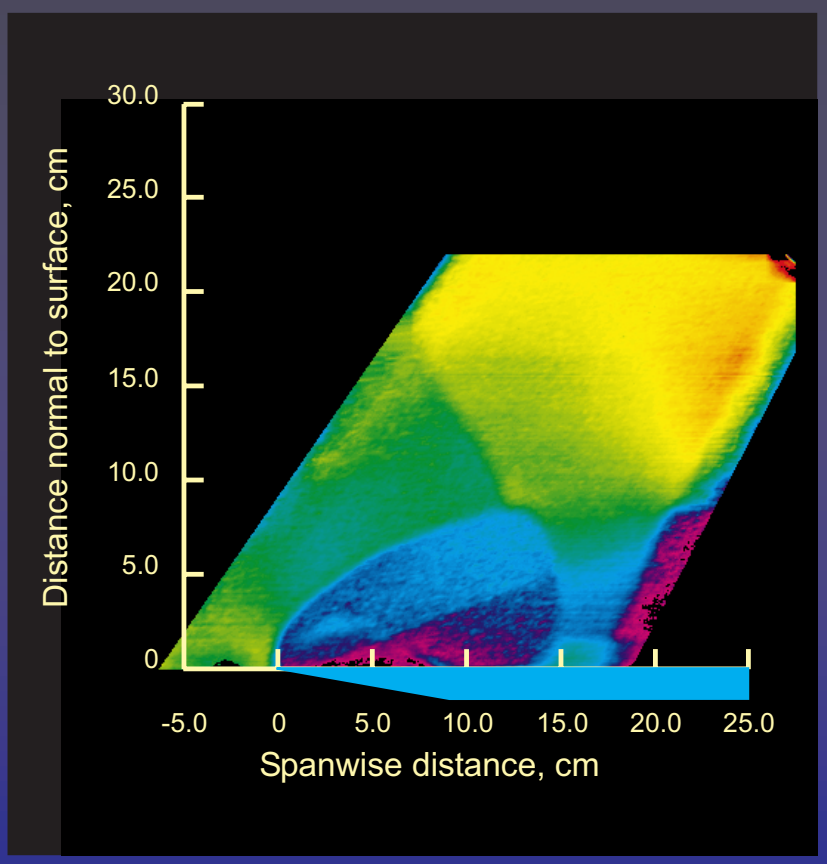

Figure 14.- Map of the velocity flow measured by the DGV of the vortical flow above a 75-degree delta wing at the 95-percent chord location at Mach 2.8. 


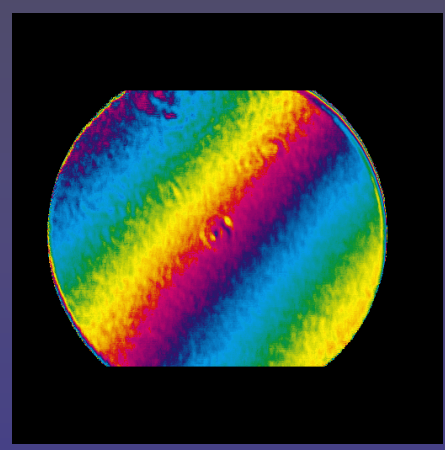

A

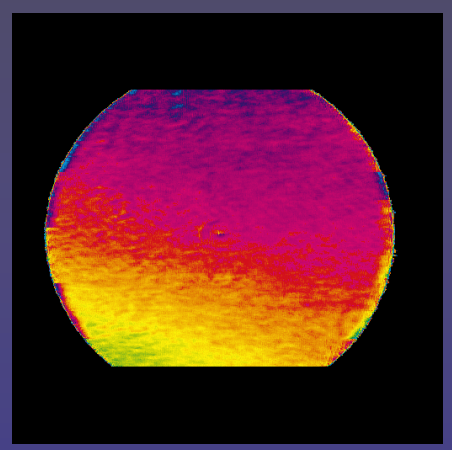

B

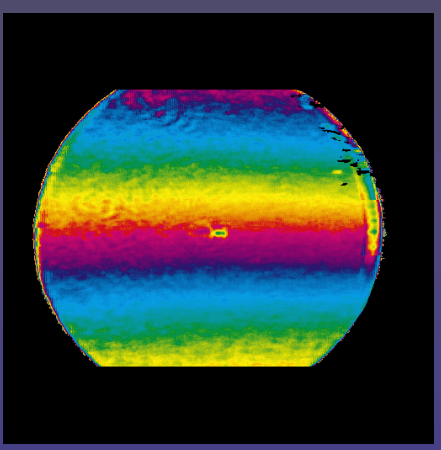

C

Figure 15.- Velocity images of a rotating wheel obtained from the three views shown in figure 5 .

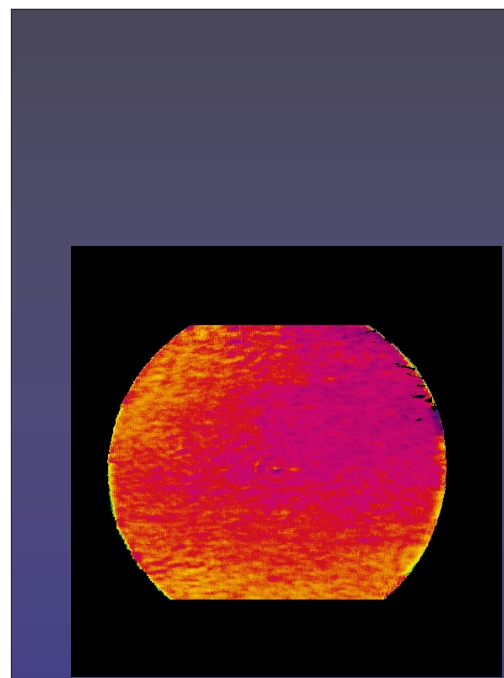

U

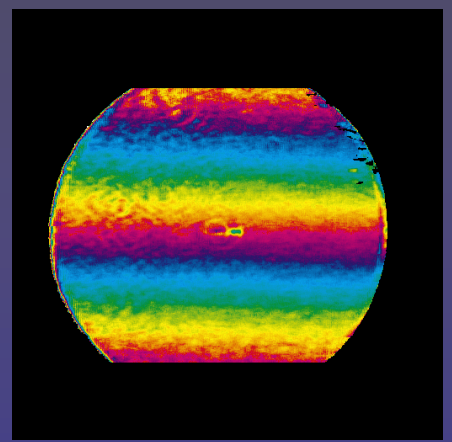

$\checkmark$

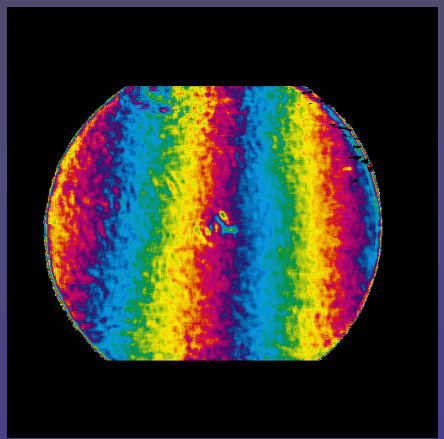

W

Figure 16.- The $u, v$, and $w$ velocity components resolved from the data images presented in figure 15 . 


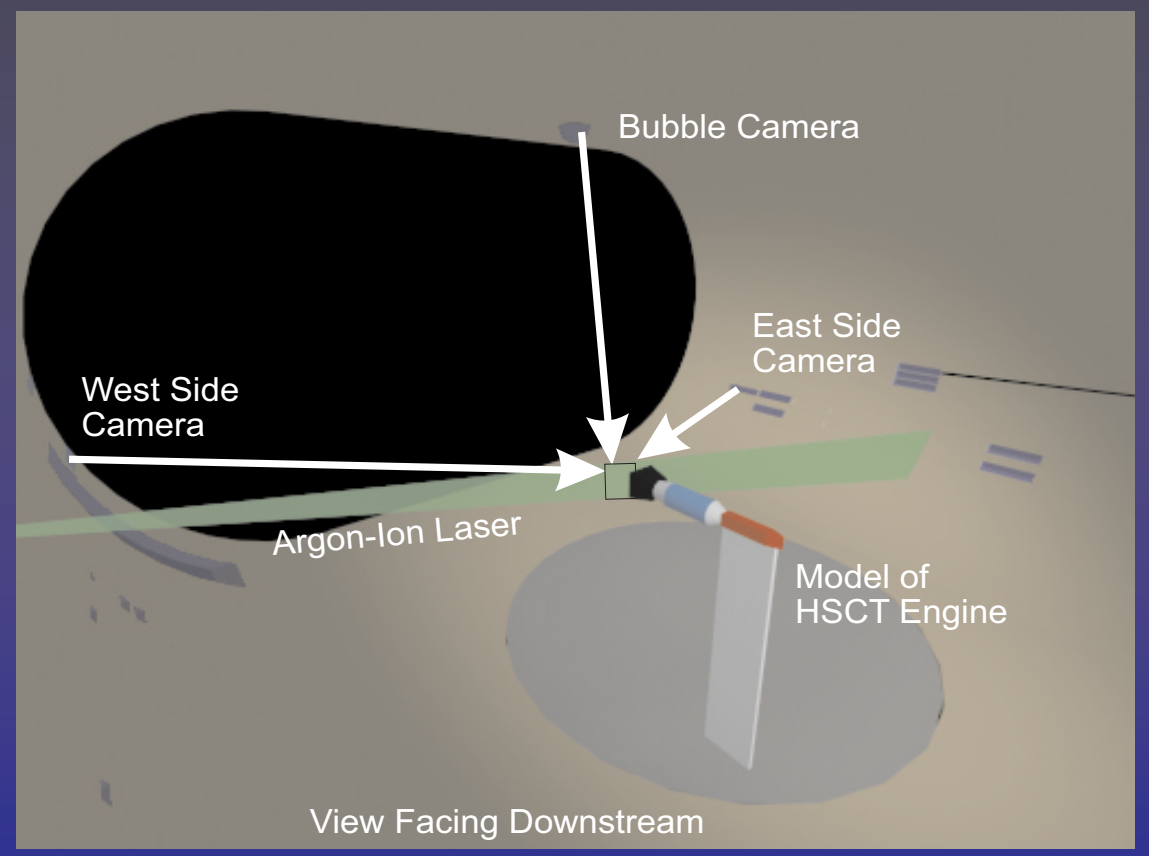

Figure 17.- Configuration of the three-component DGV optical system in the NASA Ames Research Center 40-x 80-foot Wind Tunnel to measure the flow from a high-speed jet.
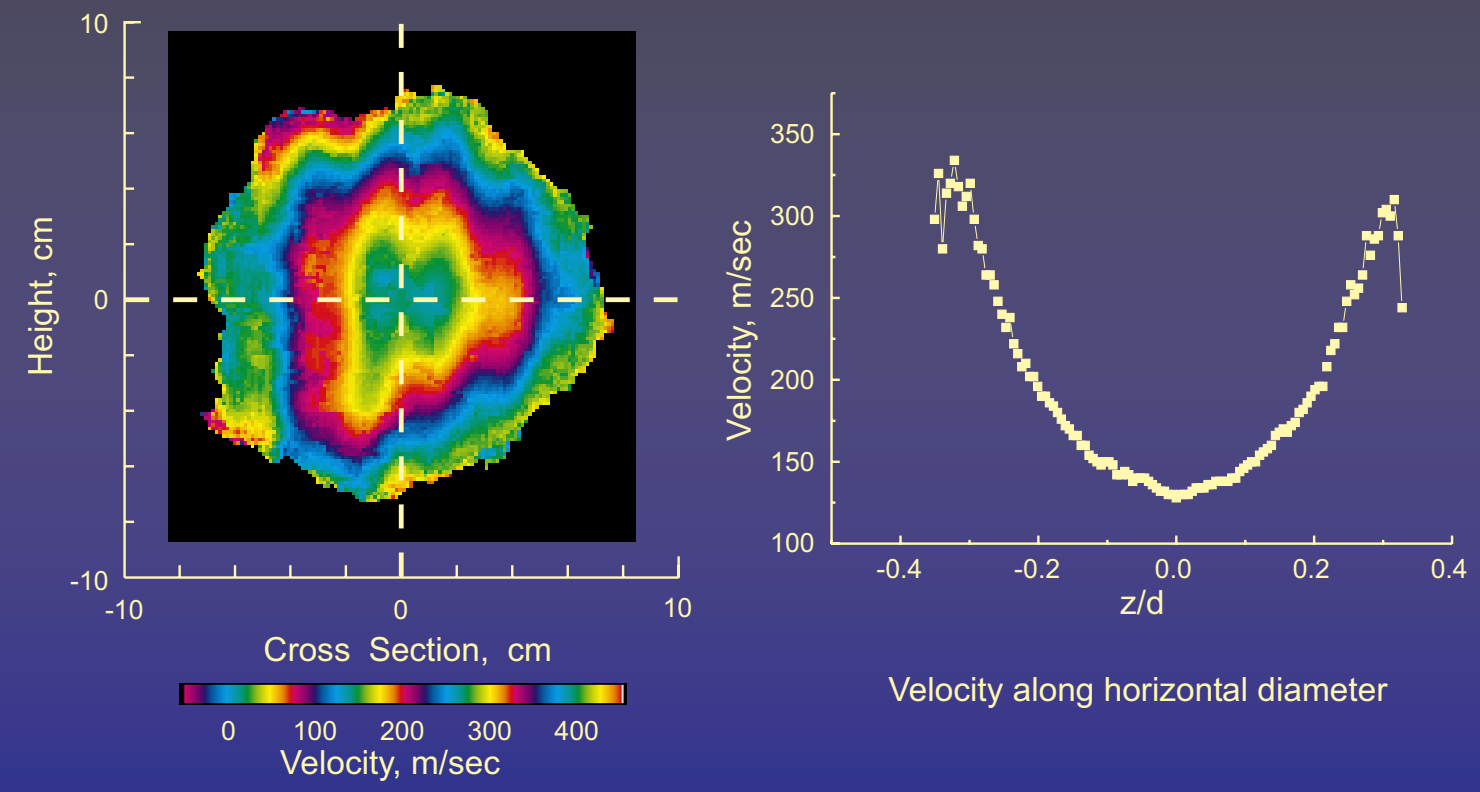

Velocity along horizontal diameter

Figure 18.- Resolved streamwise component of velocity from the high-speed jet flow operating at $463^{\circ} \mathrm{C}$ at a free stream Mach number equal to 0.15 . 